\title{
Dispersing single-walled carbon nanotubes in ionic liquids: a quantitative analysis
}

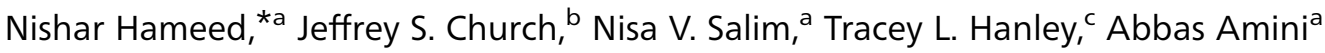 \\ and Bronwyn L. Fox ${ }^{a}$ \\ The efficiency of various ionic liquids, specifically the 1-butyl-3-methylimidazolium tetrafluoroborate, \\ hexafluorophosphate, chloride and dicyanamide salts, in dispersing single walled carbon nanotubes has \\ been examined. The SWCNTs were dispersed in the ILs at varying concentrations by grinding. All of the \\ ILs were found to be effective dispersants for nanotubes and quantitative evidences including $X$-ray \\ scattering, Raman spectroscopy and UV-visible spectroscopy unambiguously showed that BMIM[BF $F_{4}$ is \\ the most efficient IL while BMIM[DCA] is the least effective IL. A quantitative analysis of the interactions \\ between SWCNTs and ionic liquids by analyzing their scattering and spectral features is put forth.
}

Received 20th May 2013

Accepted 12th August 2013

DOI: $10.1039 / c 3 r a 42488 j$

www.rsc.org/advances

\section{Introduction}

The unique combination of outstanding mechanical, thermal, and electrical properties of CNTs makes them excellent for the fabrication of advanced structural and functional materials. ${ }^{1}$ However, the practical applications of CNTs are limited because of their extremely low solubility and poor dispersion characteristics; CNTs tend to form aggregated bundles through van der Waals tube-tube interactions. ${ }^{2}$ The approach to improve dispersion by covalent functionalization may disrupt the $\pi$-networks of CNTs and thereby reduce their inherent physical properties. ${ }^{3}$ Through non-covalent functionalization, involving the adsorption of suitable molecules, effective dispersion of CNTs can be achieved while still preserving their desired properties..$^{3-5}$ Ionic and non-ionic surfactants have been widely investigated as stabilizing agents for CNTs in various aqueous and non-aqueous media. ${ }^{6,7}$

A simple and more efficient method for dispersing CNTs has been achieved using room-temperature ionic liquids (ILs). ${ }^{8}$ Highly dispersed and stable gelatinous soft materials called 'bucky gels' were prepared by mechanically mixing SWCNTs and imidazolium ion-based ionic liquids. Bucky gels have many important applications such as the fabrication of electrochemical devices, ${ }^{9,10}$ plastic actuators, ${ }^{11,12}$ elastic conductors, ${ }^{13,14}$ and $\mathrm{CNT} /$ polymer composites. ${ }^{15,16}$ Although the preparation and applications of bucky gels were documented, a systematic study concerning the dispersion and interaction has so far not been performed. Identifying the accurate CNT dispersion in ILs

${ }^{a}$ Institute for Frontier Materials, Deakin University, Geelong, Victoria 3216, Australia. E-mail: nishar.hameed@deakin.edu.au

${ }^{b}$ CSIRO Materials Science and Engineering, Waurn Ponds, Victoria 3216, Australia

${ }^{c}$ Institute of Materials Engineering, Australian Nuclear Science and Technology Organisation Lucas Heights, New South Wales 2234, Australia would enable the optimization of various structural and functional properties of these materials. In this paper, we present a quantitative analysis of the dispersion of SWCNTs in ionic liquids with a wide range of anions by analyzing their scattering and spectral features.

\section{Experimental}

\subsection{Materials and preparation}

High purity short SWCNTs were obtained from Sigma Aldrich. The ionic liquids, 1-butyl-3-methylimidazolium chloride (Fluka), 1-butyl-3-methylimidazolium hexafluorophosphate (Sigma-Aldrich) and 1-butyl-3-methylimidazolium dicyanamide and 1-butyl-3-methylimidazolium tetrafluoroborate (both from Merck Chemicals) were used as received to simplify the process. SWCNT/IL suspensions were prepared by weighing appropriate amounts of nanotubes into a mortar and grind well with each ionic liquid for 30 minutes. Suspensions containing $0.1,0.3,0.5$ and $1 \mathrm{wt} \%$ of SWCNTs were prepared in this way.

\subsection{Characterization}

USAXS experiments were performed at the Australian Synchrotron on the ultra-small and small angle X-ray scattering beamline utilizing an undulator source that allows measurement at a very high flux to moderate scattering angles and a good flux at the minimum $q$ limit. The experiments were conducted at room temperature and the X-ray wavelength was $0.062 \mathrm{~nm}$. The intensity profiles were interpreted from the plot of scattering intensity $(I)$ versus scattering vector, $q=(4 \pi / \lambda) \sin (\theta / 2)$; where $\theta$ is the scattering angle.

Raman spectra were obtained using an inVia confocal microscope system (Renishaw, Gloucestershire, UK) with $785 \mathrm{~nm}$ excitation from a Renishaw diode laser through a $\times 20$ (0.4 na) objective. Incident laser power, as measured using an 
Ophir Nova power meter fitted with a PD300-3W head, was $2.8 \mathrm{~mW}$. For the incident rectangular beam size $(2 \mu \mathrm{m} \times 50 \mu \mathrm{m})$, a laser power density of $2.8 \mathrm{~kW} \mathrm{~cm}^{-2}$ can be calculated. Spectra were collected over the range 3199 to $100 \mathrm{~cm}^{-1}$ by averaging at least 10 scans, each with an accumulation time of 20 seconds. Eight spectra were collected from different areas of the SWCNT/ IL suspensions. The Raman shifts were calibrated using the $520 \mathrm{~cm}^{-1}$ line of a silicon wafer and the spectral resolution was $\sim 1 \mathrm{~cm}^{-1}$. Spectral manipulation was carried out using Grams AI v 9.1 (Thermo Scientific, USA). Spectral deconvolution was carried out based on peak components identified by $2^{\text {nd }}$ derivative spectra obtained using the Savitzky-Golay method. ${ }^{17}$ Fits are based on the usage of a minimal number of band components. All peaks heights were limited to the range greater than or equal to zero. In the initial fitting steps the band centers were only allowed to vary by $\pm 5 \mathrm{~cm}^{-1}$ from the frequency determined by the second derivative spectra. In the final refinements all parameters were allowed to vary unconstrained. Two point linear baselines were used throughout.

The UV-Vis spectra were measured on Perkin-Elmer Lambda 35 at $60 \mathrm{~nm} \min ^{-1}$ with $1 \mathrm{~nm}$ slits in dual beam operation. Samples were mounted as thin films between UV-rated quartz discs, differences in film thicknesses were accounted for by normalising all spectral series to the peak at $220 \mathrm{~nm}$ which is attributable to the BMIM cation which is common for all samples. IL contributions were subsequently subtracted from the spectra to show differences apparent as a result of incorporation CNTs.

Transmission electron microscopy experiments were performed on a JEOL JEM-2100 transmission electron microscope at an acceleration voltage of $100 \mathrm{kV}$. A drop of the SWCNT/IL suspension was placed onto a carbon-coated TEM copper grid. The excess sample was blotted away using a strip of filter paper. The samples were kept under vacuum for 48 hours before measurement.

\section{Results and discussion}

In order to study the dispersion and its mechanism, controlled experiments were conducted using various imidazolium ionbased ionic liquids and prepared suspensions of SWCNTs from 0.1 up to $1 \mathrm{wt} \%$ nanotube concentrations. Due to the unique structure of the ILs, the imidazolium ions have a substantial affinity for SWCNTs, and therefore, the organic salts adsorb onto the surface of the nanotubes. ${ }^{18}$ SWCNT/IL suspensions were prepared using ILs with identical 1-butyl-3-methylimidazolium $\left(\mathrm{BMIM}^{+}\right)$cation and the four different anions; tetrafluoroborate $\left[\mathrm{BF}_{4}{ }^{-}\right]$, hexafluorophosphate $\left[\mathrm{PF}_{6}^{-}\right]$, dicyanamide $\left[\left(\mathrm{CN}_{2}\right) \mathrm{N}^{-}\right]$ (DCA) and chloride $\left[\mathrm{Cl}^{-}\right]$. High purity short SWCNTs were of 1$2 \mathrm{~nm}$ in diameter and $0.5-2 \mu \mathrm{m}$ in length. The suspensions were prepared by grinding a mixture of SWNTs and IL in an agate mortar followed by storage in plastic containers. The SWCNT dispersing ability of the ionic liquids was determined visually; by keeping dispersions for several weeks they were defined as being stable if no sedimentation was present. A photograph of SWCNT suspensions in BMIM[Cl] 10 days after preparation at various nanotube concentrations is shown in Fig. 1a.
The suspensions contain isolated rigid rods or branched, ropelike overlapping SWCNT bundles and both can be observed using transmission electron microscopy (TEM). ${ }^{19}$ In this study the TEM measurements confirm that all of the investigated ILS dispersed suspensions contain debundled SWCNTs. Fig. 1b shows a TEM image of an individual SWCNT dispersed using $\operatorname{BMIM}\left[\mathrm{BF}_{4}\right]$. The gel behavior observed in these mixtures is apparently due to the formation of networks owing to the overlapping of individual SWCNTs or their bundles depending on the dispersion state. The average distance between the two neighboring contact points in the network is the mesh size $(\xi)$ (Fig. 1c). ${ }^{19}$ The microscopic dispersion of SWCNTs in ionic liquids can be studied using ultra small-angle X-ray scattering (USAXS) techniques. The concentration dependence of $\xi$ can be determined from USAXS intensity profiles to quantify the network crossover effect. The $\xi$ value is expected to decrease with increasing SWCNT concentrations. The rigid rod behavior and crossover network behavior of viscous SWCNT suspensions can be quantitatively demonstrated by careful analysis of USAXS data at various $Q$ regions.

Individual SWCNTs can be considered as one-dimensional objects and isolated CNTs or rod-like CNT bundles are expected to exhibit a $Q^{-1}$ intensity variation in the USAXS scattering profile as shown in Fig. $1 \mathrm{~d} .^{20}$ Alternately the scattering intensity of poorly dispersed CNT aggregates exhibit a $Q^{-2}$ behavior. Fig. 2 shows the USAXS profiles of SWCNT suspensions stabilized by different ILs. The $Q^{-1}$ behavior is apparent in all the scattering profiles indicating the dispersion of nanotubes down to individual levels. Moreover all profiles show an upturn in scattering at $Q<0.01 \AA^{-1}$ which is due to the presence of aggregated nanotubes. ${ }^{21}$ The rigid $\operatorname{rod}\left(Q^{-1}\right)$ and network structure $\left(Q^{-2}\right)$ contributions are highlighted through straight line power law fitting in each figure and $Q_{c}$ represents the crossover point.
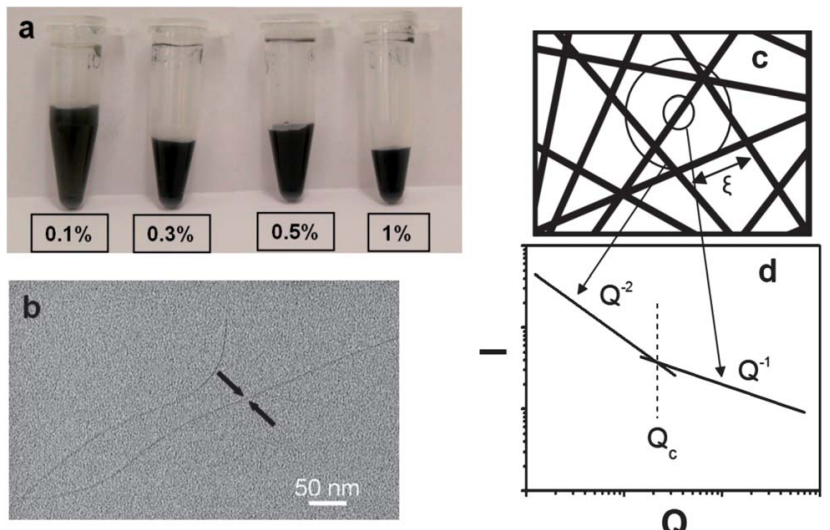

Fig. 1 (a) A photograph depicting SWCNT suspensions in BMIM[CI] at different CNT concentrations. (b) TEM image of an individually dispersed SWCNT in BMIM $\left[\mathrm{BF}_{4}\right]$. (c) Possible structure of a SWCNT dispersion, $\xi$ is the crossover point that gives the average distance between the intersections of the crosslinked network (the small circle in the picture represents the individual nanotube and big circle is the network structure). (d) The typical scattering profile model illustrating the crossover point in a CNT suspension. ${ }^{19}$ 

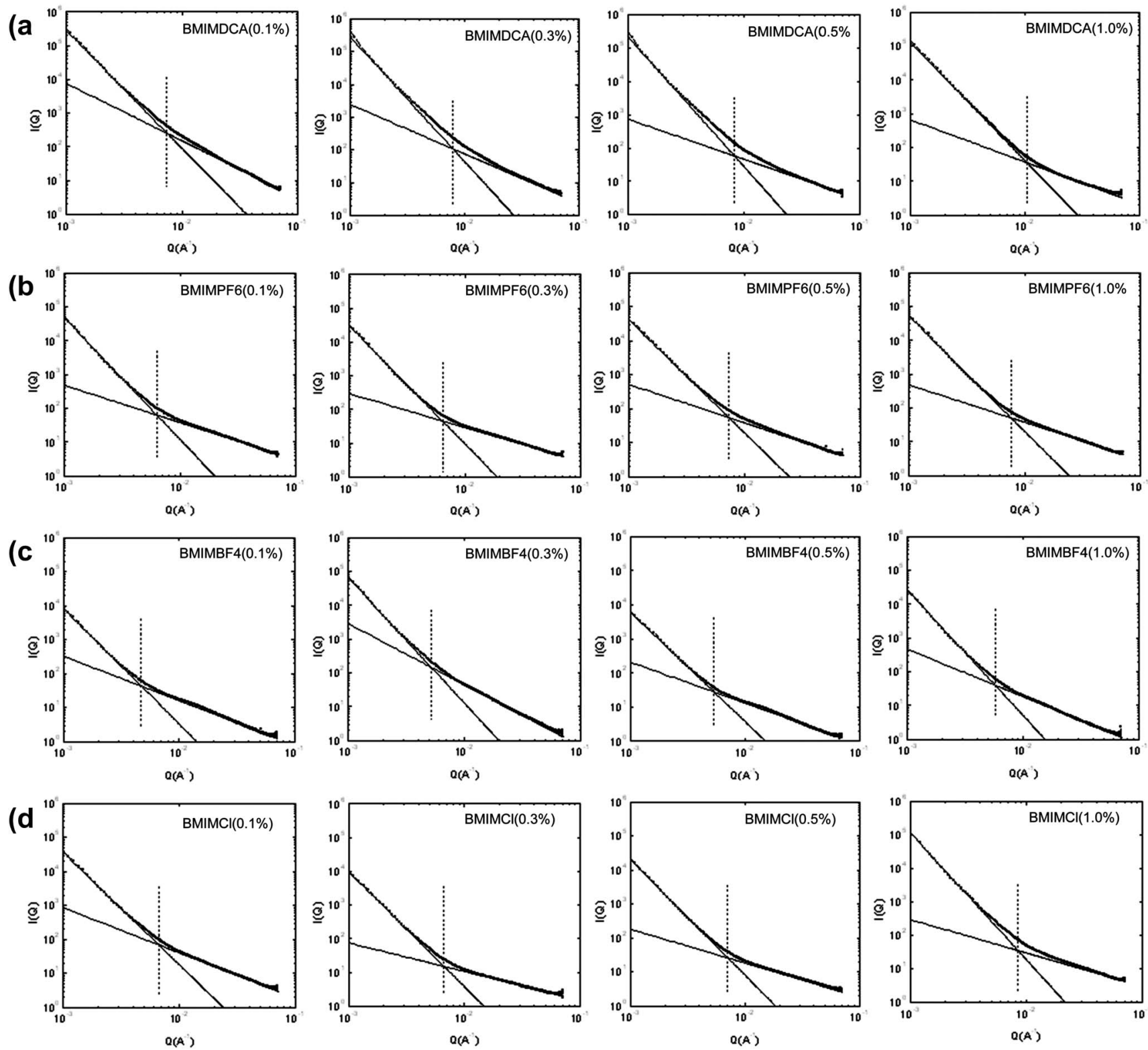

Fig. 2 USAXS profiles of IL/SWCNT suspensions at various nanotube concentrations. (a) BMIM[DCA]/SWCNT; (b) BMIM[PF 6 /SWCNT; (c) BMIM[BF $4 / S W C N T$ and (d) BMIM[CI]/SWCNT. The solid lines represent the $Q^{-1}$ to $Q^{-2}$ power law behaviors and the vertical dotted lines represent the crossover points. The $\xi$ values are calculated from the crossover points and are given in Table 1.

USAXS profiles are often distorted by the presence of other scattering species. It is reported that the intensity profiles of surfactant assisted SWCNT dispersions show a broad correlation peak at around $Q=0.06 \AA^{-1}$ due to the formation of spherical micelles. ${ }^{22}$ Moreover in surfactant systems one must subtract the contribution due to free surfactant micelles from the total scattering intensity in order to get the actual contribution of nanotube-surfactant complexes. These effects are not issues in the present study as the ILs do not form micelles or other aggregates. Also the scattering due to the ionic liquid themselves are relatively weak compared to the scattering of the nanotubes and therefore it has essentially no effect both qualitatively and quantitatively on the total scattering of ionic liquid-SWCNT complex.
The mesh size, $\xi$ gives the average distance between the SWCNTs in the network and was determined by the crossover point of the $Q^{-1}$ and $Q^{-2}$ power law behaviors $\left(\xi=2 \pi / Q_{c}\right)$. The calculated values of $\xi$ for the suspensions are given in Table 1 . Ideally, the $\xi$ value decreases with increase in the concentration of nanotubes in the suspension indicating that the nanotubes are getting closer to each other. Table 1 presents the dispersive abilities of the individual ILs as determined from the USAXS experiments. Among the four imidazolium based ILs, BMIM$\left[\mathrm{BF}_{4}\right]$ shows the highest $\xi$ values at any SWCNT concentration. This demonstrates the comparatively better capability of BMIM$\left[\mathrm{BF}_{4}\right]$ in dispersing nanotubes.

It has been noted that Raman spectroscopy, in particular, the frequencies of the radial breathing (RBM) modes, $\omega_{\mathrm{RBM}}$, should 


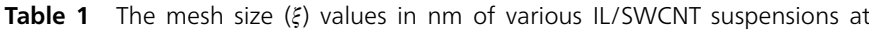
different concentrations of nanotubes

$\xi$ at different SWCNT loading $(\mathrm{nm})$

\begin{tabular}{lcccc} 
IL & $0.1 \mathrm{wt} \%$ & $0.3 \mathrm{wt} \%$ & $0.5 \mathrm{wt} \%$ & $1 \mathrm{wt} \%$ \\
\hline $\mathrm{BMIM}\left[\mathrm{BF}_{4}\right]$ & 134 & 122 & 117 & 110 \\
$\mathrm{BMIM}\left[\mathrm{PF}_{6}\right]$ & 100 & 98 & 86 & 83 \\
BMIM[Cl] & 94 & 90 & 88 & 74 \\
BMIM[DCA] & 84 & 79 & 76 & 59
\end{tabular}

be sensitive to the state of aggregation of single walled carbon nanotubes. A number of theories have been put forth and a significant amount of experimental and theoretical work is in the literature. ${ }^{23-28}$ While Jorio and co-workers ${ }^{25}$ suggest that the shift in RBM frequencies due to de-bundling of SWCNTs in the diameter range of 1-2 $\mathrm{nm}$ should be of the order of $4 \mathrm{~cm}^{-1}$ (higher in frequency for $1 \mathrm{~nm}$ tubes and lower for $2 \mathrm{~nm}$ tubes), Sauvajol et al. ${ }^{27}$ suggests a more significant $10 \mathrm{~cm}^{-1}$ shift to lower frequency upon de-bundling, irrespective of tube diameter. Husanu and co-worker, ${ }^{24}$ in a resonance Raman study of aqueous surfactant dispersed SWCNTs using $1064 \mathrm{~nm}$ excitation reported a $10 \mathrm{~cm}^{-1}$ shift to lower frequency upon debundling. In contrast, other workers ${ }^{26-28}$ found no effect on the RBMs upon de-bundling with either aqueous surfactants or ILs.

The low wavenumber region of a typical Raman spectrum obtained from the SWCNTs is shown in Fig. 3a. Based on the
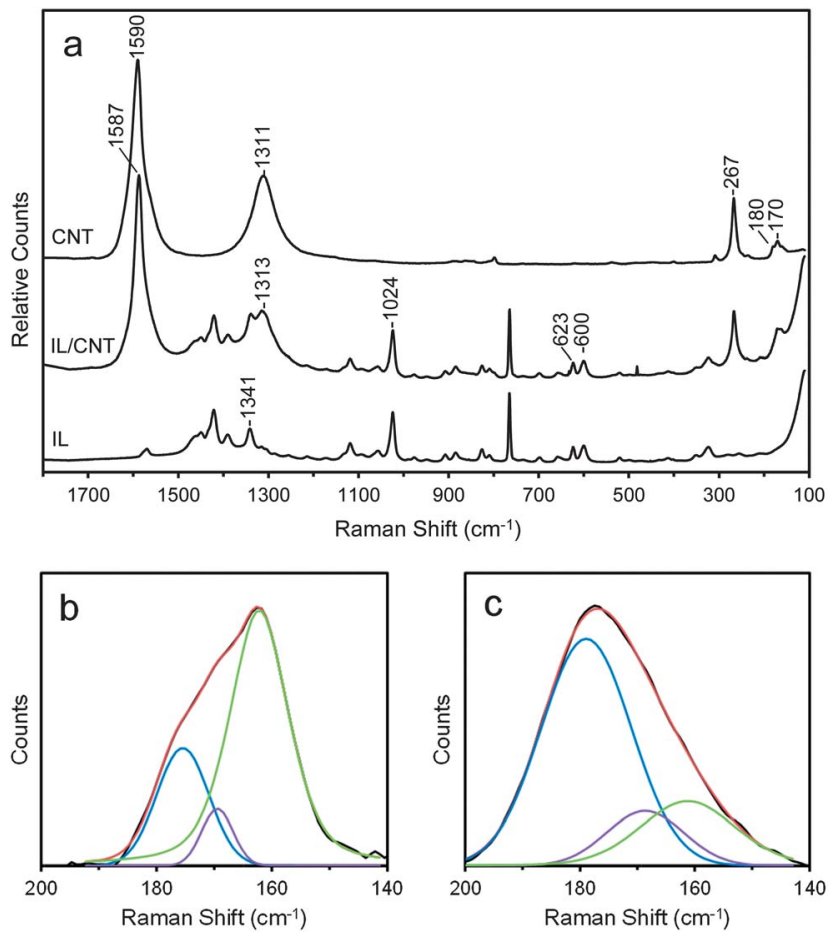

Fig. 3 (a) Typical low frequency Raman spectrum obtained from the SWCNTs, a 1 wt $\%$ SWCNT suspension in BMIM[BF $\left.F_{4}\right]$ and pure BMIM[BF $\left.F_{4}\right]$. Deconvolution of the low frequency RBM for SWCNTs dispersed in (b) BMIM[CI] and (c) BMIM[DCA]. The red trace is the actual data while the black trace represents the sum of the components. experimental conditions used and considering a typical Kataura plot (energy, $E_{\mathrm{ii}}$ verse nanotube diameter, $d_{\mathrm{t}}$ ), ${ }^{25}$ semiconducting and metallic CNTs with the energy transitions $E_{22}^{\mathrm{S}}$ and $E_{11}^{\mathrm{M}}$ would are expected to be in resonance. The $\mathrm{G}$ and $\mathrm{D}$ bands are consistently observed at 1590 and $1311 \mathrm{~cm}^{-1}$, respectively and two RBMs are apparent in the 300 to $100 \mathrm{~cm}^{-1}$ range. The most intense and highest frequency RBM is observed as a single sharp peak with a maximum consistently observed within $1 \mathrm{~cm}^{-1}$ of $267 \mathrm{~cm}^{-1}$. The lower frequency RBM is in comparison, quite weak. Consistent features include an intensity maximum at $\sim 170 \mathrm{~cm}^{-1}$ and weaker components near 180 and $159 \mathrm{~cm}^{-1}$.

Typical spectra obtained from IL dispersed SWCNTs and the corresponding pure IL, are also shown in Fig. 3. The same frequencies for the $\mathrm{G}$ band $\left(1587 \mathrm{~cm}^{-1}\right)$, D band $\left(1313 \mathrm{~cm}^{-1}\right)$ and RBM $\left(267 \mathrm{~cm}^{-1}\right)$ were observed irrespective of the IL used. These frequencies are slightly shifted from those observed in the spectra obtained from the pure CNTs. The width of the G band has also become narrower upon dispersion suggesting a more uniform CNT environment. The frequencies of the IL bands observed in the spectra obtained from the SWCNT suspension were found, in general, to be in excellent agreement with those of the pure ILs. The relative intensity ratios of the bands at $\sim 600$ and $623 \mathrm{~cm}^{-1}{ }^{29}$ suggest that the anti-anti, gauche-anti conformational equilibrium of the substituted imidazolium cations of the $\left[\mathrm{Cl}^{-}\right],\left[\mathrm{BF}_{4}{ }^{-}\right]$and $\left[\mathrm{PF}_{6}{ }^{-}\right]$salts are very similar ( 1.2). Only relatively small changes in this ratio $(<10 \%)$, and no changes in the anion bands were observed in the spectra obtained from these SWCNT/IL suspensions. These results are consistent with those reported by Wang et al. ${ }^{28}$ who suggest that the ILs and CNTs interact by weak van der Waals interactions and not by "cation- $\pi$ " interactions as previously assumed. Based on the 598 and $624 \mathrm{~cm}^{-1}$ ratio, the conformational structure of the [DCA] salt is quite different, largely gauche-anti (16.2). The change in this ratio in the CNT dispersion was also less than $10 \%$ but a $2 \mathrm{~cm}^{-1}$ shift to lower frequency was observed for the $\mathrm{C} \equiv \mathrm{N}$ stretching vibration suggesting that these groups are less constrained.

It is difficult to impossible to assess changes to the low frequency RBM due to overlap with bands from the ILs or the onset of the ILs Raleigh line. In an attempt to isolate the features of this RBM, the spectra obtained from the corresponding pure ILs was subtracted from the spectra obtained from the suspensions with the scale factor being determined by minimizing the residual of the $1024 \mathrm{~cm}^{-1}$ IL imidazole $\delta$ ring mode. ${ }^{30}$ This mode is common to all IL spectra and does not overlap with any SWCNT features. In contrast to the higher frequency $267 \mathrm{~cm}^{-1}$ RBM, the shape of the lower frequency $\left(170 \mathrm{~cm}^{-1}\right)$ mode was found to change as a function of IL. This is consistent with the results that Husanu and co-workers ${ }^{24}$ found in their study of aqueous surfactant dispersions of SWCNTs.

Deconvolution of the low frequency RBMs were carried out based on the results of the $2^{\text {nd }}$ derivative spectra determined for the spectral subtractions. Three peak components were identified and deconvolutions for the $1 \mathrm{wt} \%$ suspensions of the SWCNTs in BMIM[Cl] and BMIM[DCA] are shown as Fig. 3(b) and (c), respectively. The deconvolution results for all $\mathrm{IL} /$ SWCNTs suspensions in this study and their analysis based on 
Table 2 The low frequency RBM component parameters obtained for the various IL/SWCNT suspensions

\begin{tabular}{lllll}
\hline $\begin{array}{l}\mathrm{IL} / \mathrm{SWCNT} \\
\text { dispersion }\end{array}$ & $\begin{array}{l}\omega_{\mathrm{RBM}} \\
\text { isolated }\left(\mathrm{cm}^{-1}\right)\end{array}$ & $\begin{array}{l}\omega_{\mathrm{RBM}} \\
\left(\mathrm{cm}^{-1}\right)\end{array}$ & $\begin{array}{l}\omega_{\mathrm{RBM}} \\
\text { bundled }\left(\mathrm{cm}^{-1}\right)\end{array}$ & $\begin{array}{l}I_{\text {Isolated }} \\
I_{\text {Bundled }}\end{array}$ \\
\hline $\mathrm{BMIM}\left[\mathrm{BF}_{4}\right]$ & 166 & 171 & 178 & 3.7 \\
$\mathrm{BMIM}[\mathrm{Cl}]$ & 162 & 170 & 176 & 2.1 \\
$\mathrm{BMIM}\left[\mathrm{PF}_{6}\right]$ & 163 & 169 & 175 & 2.1 \\
$\mathrm{BMIM}[\mathrm{DCA}]$ & 166 & 168 & 179 & 0.3 \\
\hline
\end{tabular}

the approach of Husanu et $a .^{24}$ are summarized in Table 2. Briefly, the two RBM components observed near 164 and $178 \mathrm{~cm}^{-1}$ are attributed to isolated and bundled SWCNTS, respectively. A third component was observed near $170 \mathrm{~cm}^{-1}$, which consistently contributed of the order of $10 \%$ of the total $\mathrm{RBM}$ area. Based on the ratio of these bands, $\mathrm{BMIM}\left[\mathrm{BF}_{4}\right]$ clearly has the best dispersing ability while BMIM[DCA] has the worst.

The dispersion of SWCNTs in the various ILs was further examined using UV-Vis spectroscopy. It was reported that bundles SWCNTs are barely active in the 200-800 $\mathrm{nm}$ region of the UV-Vis spectra as their photoluminescence is quenched. ${ }^{31}$ However, individually dispersed nanotubes exhibit strong absorption bands in this region. ${ }^{32}$ Fig. 4 shows the Vis spectra of SWCNT/IL suspensions. From the figure, it can be confirmed that $\mathrm{BMIM}\left[\mathrm{BF}_{4}\right]$ shows the highest absorption intensity slope in the high frequency region followed by BMIM[PF 6$]$ and BMIM$[\mathrm{Cl}]$. However the difference between the absorption of BMIM$\left[\mathrm{PF}_{6}\right]$ and $\mathrm{BMIM}[\mathrm{Cl}]$ is nominal. Moreover, BMIM[DCA] shows the least intense absorption compared to other three ILs indicating its comparatively low efficiency for the CNT dispersion.

It has been reported that the dispersion of SWCNTs in IL occurs by van der Waals forces which effectively prevent the detached nanotubes from re-bundling. ${ }^{28}$ It was further suggested that the SWCNT/IL dispersion is not significantly influenced by the local structure of the IL cations, however there is

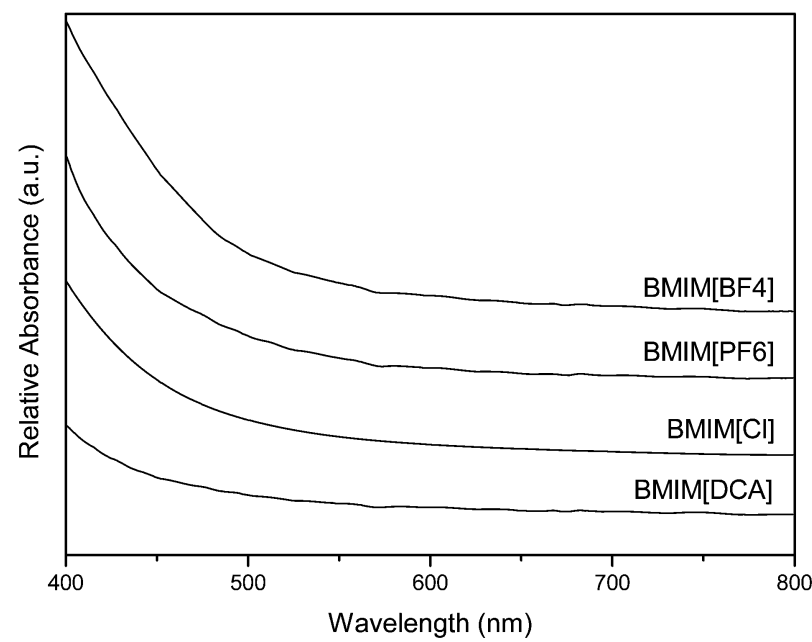

Fig. 4 The absorption spectra in the $400-800 \mathrm{~nm}$ region of $1 \mathrm{wt} \%$ SWCNTs dispensed in various ILs. an interfacial effect of anions on the surrounding nanotubes and it can be assumed that this effect may vary with the nature of the anions. All the above mentioned evidence imply that $\mathrm{BMIM}\left[\mathrm{BF}_{4}\right]$ has the highest and $\mathrm{BMIM}[\mathrm{DCA}]$ has the lowest dispersing power. The $\mathrm{BMIM}[\mathrm{Cl}]$ and $\mathrm{BMIM}\left[\mathrm{PF}_{6}\right]$ have intermediate effects on suspension. This is largely consistent with the results determined by USAXS, Raman spectroscopy and visible spectroscopy in this study. The Raman and visible spectra suggest that $\mathrm{BMIM}[\mathrm{Cl}]$ and $\mathrm{BMIM}\left[\mathrm{PF}_{6}\right]$ are very similar in their effect while the USAXS results indicate that the $\left[\mathrm{PF}_{6}{ }^{-}\right]$ salt is a slightly better dispersant than the $\left[\mathrm{Cl}^{-}\right]$salt.

\section{Conclusions}

We have examined the efficiency of various imidazolium ILs in dispersing SWCNTs. The ILs were all found to be effective dispersants for nanotubes and quantitative evidences unambiguously show that $\mathrm{BMIM}\left[\mathrm{BF}_{4}\right]$ is the most efficient IL while $\mathrm{BMIM}[\mathrm{DCA}]$ is the least effective IL in dispersing nanotubes. The ability to disperse nanotubes as individual tubes with ILs while retaining their excellent intrinsic properties may enable greater use of the interesting chemical and physical properties of nanotubes. Moreover, by taking advantage of the high thermal stability of ILs together with their outstanding ionic conductivity, a clear strategy for the fabrication of advanced materials is provided.

\section{Acknowledgements}

SAXS measurements were undertaken at the Australian Synchrotron, Victoria, Australia, and the authors thank Dr Nigel Kirby and Dr Adrian Hawley for their assistance. We also thank the Australian Institute of Nuclear Science and Engineering (AINSE) Ltd for a research grant (ALNGRA 11157) enabling the SAXS measurements.

\section{References}

1 R. H. Baughman, A. A. Zakhidov and W. A. D. Heer, Science, 2002, 297, 787-792.

2 D. Tasis, N. Tagmatarchis, A. Bianco and M. Prato, Chem. Rev., 2006, 106, 1105-1136.

3 A. Star, Y. Liu, K. Grant, L. Ridvan, J. F. Stoddart, D. W. Steuerman, M. R. Diehl, A. Boukai and J. R. Heath, Macromolecules, 2003, 36, 553-560.

4 R. J. Chen, S. Bangsaruntip, K. A. Drouvalakis, N. W. S. Kam, M. Shim, Y. Li, W. Kim, P. J. Utz and H. Dai, Proc. Natl. Acad. Sci. U. S. A., 2003, 100, 4984-4989.

5 X. F. Zhang, T. Liu, T. V. Sreekumar, S. Kumar, V. C. Moore, R. H. Hauge and R. E. Smalley, Nano Lett., 2003, 3, 12851288.

6 B. G. Cousins, A. K. Das, R. Sharma, Y. Li, J. P. McNamara, I. H. Hillier, I. A. Kinloch and R. V. Ulijn, Small, 2009, 5, 587-590.

7 N. R. Tummala, B. H. Morrow, D. E. Resasco and A. Striolo, ACS Nano, 2010, 4, 7193-7204. 
8 T. Fukushima, A. Kosaka, Y. Ishimura, T. Yamamoto, T. Takigawa, N. Ishii and T. Aida, Science, 2003, 300, 20722074.

9 E. Miyako, H. Nagata, R. Funahashi and T. Hirotsu, ChemSusChem, 2009, 2, 740-742.

10 L. Zhao, Y. Li, Z. Liu and H. Shimizu, Chem. Mater., 2010, 22, 5949-5956.

11 T. Fukushima, K. Asaka, A. Kosaka and T. Aida, Angew. Chem., Int. Ed., 2005, 44, 2410-2413.

12 K. Mukai, K. Asaka, T. Sugino, K. Kiyohara, I. Takeuchi, N. Terasawa, D. N. Futaba, K. Hata, T. Fukushima and T. Aida, Adv. Mater., 2009, 21, 1582-1585.

13 T. Sekitani, H. Nakajima, H. Maeda, T. Fukushima, T. Aida, K. Hata and T. Someya, Nat. Mater., 2009, 8, 494-499.

14 T. Sekitani, Y. Noguchi, K. Hata, T. Fukushima, T. Aida and T. Someya, Science, 2008, 321, 1468-1472.

15 N. Liu, F. Luo, H. Wu, Y. Liu, C. Zhang and J. Chen, Adv. Funct. Mater., 2008, 18, 1518-1525.

16 H. Zhang, Z. Wang, Z. Zhang, J. Wu, J. Zhang and J. He, Adv. Mater., 2007, 19, 698-704.

17 A. Savitzky and M. J. E. Golay, Anal. Chem., 1964, 36, 16271639.

18 D. A. Crescenzo, M. Aschi, E. D. Canto, S. Giordani, D. Demurtas and A. Fontana, Phys. Chem. Chem. Phys., 2011, 13, 11373-11383.

19 L. A. Hough, M. F. Islam, B. Hammouda, A. G. Yodh and P. A. Heiney, Nano Lett., 2006, 6, 313-317.

20 W. Zhou, Chem. Phys. Lett., 2004, 384, 185-189.
21 K. Yurekli, C. A. Mitchell and R. Krishnamoorti, J. Am. Chem. Soc., 2004, 126, 9902-9903.

22 H. Wang, W. Zhou, D. L. Ho, K. I. Winey, J. E. Fischer, C. J. Glinka and E. K. Hobbie, Nano Lett., 2004, 4, 17891793.

23 D. A. Heller, P. W. Barone, J. P. Swanson, R. M. Mayrhofer and J. P. C. B. M. S. Strano, J. Phys. Chem. B, 2004, 108, 6905-6909.

24 M. Husanu, M. Baibarac, N. Preda and J. O. A. M. I. Baltog, J. Optoelectron. Adv. Mater., 2008, 10, 1722-1726.

25 A. Jorio, M. A. Pimenta, A. G. S. Filho, R. Saito, G. Dresselhaus and N. J. P. M. S. Dresselhaus, New J. Phys., 2003, 5, 139.

26 V. C. Moore, M. S. Strano, E. H. Haroz, R. H. Hauge, R. E. Smalley, J. Schmidt and Y. Talmon, Nano Lett., 2003, 3, 1379-1382.

27 J. L. Sauvajol, E. Anglaret, S. Rols and L. Alvarez, Carbon, 2002, 40, 1697-1714.

28 J. Wang, H. Chu and Y. Li, ACS Nano, 2008, 2, 2540-2546.

29 R. W. Berg, in Ionic Liquids in Chemical Analysis, ed. M. Koel, CRC Press, Boca Raton, USA, 2008, ch. 12.

30 D. A. Carter, J. E. Pemberton and K. J. Woelfel, J. Phys. Chem. B, 1998, 102, 9870-9880.

31 J. S. Laurent, C. Voisin, G. Cassabois, C. Delalande, P. Roussignol, O. Jost and L. Capes, Phys. Rev. Lett., 2003, 90, 057404.

32 R. Saito, M. F. G. Dresselhaus and M. S. Dresselhaus, Appl. Phys. Lett., 1992, 60, 2204-2206. 